\title{
Matrix toughness transfer and fibre bridging laws in acrylic resin based CF composites
}

\author{
T. Pini ${ }^{\mathrm{a}, *}$, F. Briatico-Vangosa ${ }^{\mathrm{a}}$, R. Frassine ${ }^{\mathrm{a}}$, M. Rink ${ }^{\mathrm{a}}$ \\ ${ }^{a}$ Politecnico di Milano, Department of Chemistry, Materials and Chemical Engineering "Giulio Natta”, Piazza \\ Leonardo da Vinci 32, I-20133, Milano, Italy
}

\begin{abstract}
The interlaminar fracture toughness, $G_{I c}$, of composite laminates produced by infusion moulding adopting two thermoplastic acrylic matrices, one plain and one rubber toughened, and continuous unidirectional carbon fibres as reinforcement was studied. For the composite with the plain matrix, fracture was dominated by the failure of the fibre-matrix interface. For the composite with the toughened matrix, it was verified that matrix toughness cannot be fully exploited due to the interaction of the fibres with the development of the process zone. A fibre bridging model based on experimentally derived cohesive zone laws allowed inferring that the fracture process occurs within the matrix.

Keywords:

matrix toughness transfer, digital image correlation, thermoplastic composites, fibre bridging, cohesive laws
\end{abstract}

\footnotetext{
*Corresponding author

Email address: tommaso.pini@polimi.it (T. Pini)
} 


\begin{tabular}{|c|c|}
\hline \multicolumn{2}{|c|}{ Nomenclature } \\
\hline$a$ & crack length \\
\hline$a_{0}$ & initial crack tip position \\
\hline$\dot{a}$ & crack propagation speed \\
\hline E & Young's modulus \\
\hline$G$ & shear modulus \\
\hline$G_{I c}$ & interlaminar mode I fracture toughness \\
\hline$G_{I c, \text { init }}$ & interlaminar mode I fracture toughness at crack initiation \\
\hline$G_{I c, p r o p}$ & interlaminar mode I fracture toughness during propagation \\
\hline$J$ & J-integral \\
\hline$J_{\text {tip }}$ & critical J-integral value at crack tip \\
\hline $\log$ & logarithm to the base 10 \\
\hline$\ell_{c, F B}$ & critical length of the bridging zone \\
\hline$N$ & density of bridging fibres \\
\hline$t_{\text {init }}$ & crack initiation time \\
\hline$T$ & temperature in Celsius degrees \\
\hline$W_{B}$ & recoverable energy in the bridging zone \\
\hline$\delta$ & crack opening \\
\hline$\delta_{c, F B}$ & critical value of crack opening for fibre bridging \\
\hline$\Gamma_{I}$ & peeling fracture resistance \\
\hline$\sigma$ & crack surface traction \\
\hline$\sigma_{L}$ & stress exerted by the bridging ligaments \\
\hline$C T O D$ & Crack Tip Opening Displacement \\
\hline DCB & Double Cantilever Beam \\
\hline DIC & Digital Image Correlation \\
\hline $\mathrm{E}$ & Elium $^{\circledR}$ based composite \\
\hline EI & Elium Impact ${ }^{\circledR}$ based composite \\
\hline
\end{tabular}




\section{Introduction}

The fracture resistance of a polymeric composite material is related to several aspects such lay-up sequence, production technique, loading mode, matrix/fibre interfacial properties and environmental conditions. Delamination or interlaminar crack propagation is a key aspect to consider when designing with composite materials, especially if a damage tolerant approach is pursued. The resistance of a material to the growth of a crack between two adjacent plies can be quantified by the mode I interlaminar fracture toughness $G_{I c}$. Although the contributions to interlaminar fracture toughness of a composite are numerous, it is widely accepted that an important role is that played by the matrix and by its ability to transfer its toughness into the laminate $[1,2]$. The major limiting factor to the full development of the process zone of the matrix is due to the constraint of the fibres in the adjacent layers of the laminate. Generally speaking, brittle matrices can fully transfer their toughness into the composite, while the efficiency of transfer for ductile matrices can be significantly lower $[1,3,2,4,5,6]$. In any case such conclusions cannot be generalized since the fracture toughness of polymeric materials is affected by their viscoelastic behaviour and may vary significantly depending on loading history (rate) and temperature. Further, the composites' delamination toughness may also be affected by fibre-related mechanisms such as fibre breaking, fibre pull-out/debonding and fibre bridging. These mechanisms take place in a fibre bridging zone, in which the intact fibres that bridge the crack surfaces exert a closing pressure. This gives rise to growing R-curves, i.e. fracture toughness increases as crack length increases. When the size of the bridging zone exceeds the thickness of the specimen, the R-curve is not a material property anymore $[7,8,9,10]$. In this case, quantification of the effect of fibre-related mechanisms for design purposes requires the modelling of the bridging zone with a cohesive law, i.e. a relationship between the crack opening displacement and the local bridging stress [7, 8, 11].

In previous works $[12,13]$ the fracture behaviour of two thermoplastic acrylic resins, one plain and one rubber toughened, and their relevant composites has been investigated at different temperatures and displacement rates. The main conclusions drawn were:

- As for the matrices, the rate and temperature dependence of the plain resin fracture behaviour is well described by viscoelastic fracture theories while for the toughened resin 
fracture behaviour is dominated by the change in deformation mechanism caused by rate and temperature.

- Regarding the relevant composites, the contribution of the matrix was deduced from fracture toughness at crack initiation while the toughness increase due to fibre related mechanisms was qualitatively evaluated from toughness during crack propagation. In the case of the plain resin based composite, toughness does not depend much on rate or temperature neither at initiation nor during propagation and it is determined by the failure of the weak fibre matrix interface. In the case of the toughened resin, it was possible to deduce that the matrix toughness is not completely transferred into the composite and the fibre related mechanisms give a large contribution and shows the same rate and temperature dependence as the neat relevant resin.

In the present work, further investigation on the same composites studied in [13] was carried out to better understand (i) the transfer of matrix toughness into the composite considering the interaction between the fibres and the development of the process zone in the composite and (ii) the fibre-related mechanisms determining experimentally a cohesive law and applying a literature model [8] for the fibre bridging mechanism.

\section{Materials and methods}

\subsection{Materials and specimens preparation}

The materials under study were two composites produced by infusion moulding. The matrices were two acrylic thermoplastic resins produced by Arkema, one plain, Elium ${ }^{\circledR}$ and one toughened, Elium Impact ${ }^{\circledR}$, made of the same resin with the addition of a $10 \mathrm{wt} \%$ of a block copolymer, Nanostrength ${ }^{\circledR}$, which produces rubbery inclusions of dimensions under $50 \mathrm{~nm}$. In both cases, unidirectional continuous T700 $12 \mathrm{~K}$ carbon fibres were used as reinforcement phase and the relevant composites are named E and EI respectively. A $13 \mu \mathrm{m}$ thick release film was placed at the laminate midplane during infusion to produce an initial delamination.

After infusion moulding, the plates of both composites underwent a thermal treatment: the conditions were the same used to produce neat resin samples in [12] and the same composites in [13] in 
order to have comparable results. The fibre weight content was measured through a resin burn-off method and then the fibre volume fraction was evaluated from the densities of fibres and matrices which were measured as well. It resulted to be around $60 \%$ for both composites. After infusion moulding and thermal treatment took place, double cantilever beams specimens (DCB) (Fig. 1) were cut from the plates. Specimens were prepared in compliance with ISO 15024:2001 standard [14]. The dimensions were 190x20x5 mm and 190x20x10 mm, for E and EI composites respectively. The thickness adopted, slightly larger than that suggested by the standard, was necessary to avoid fibre kinking and arm breakages, observed in preliminary tests. An initial delamination was introduced in the specimens as prescribed by the standard. One side of the specimens was painted white to enhance contrast and facilitate the individuation of the crack tip. A fine black speckle pattern was sprayed in a region $2-3 \mathrm{~cm}$ long in front of the initial delamination, to perform Digital Image Correlation (DIC) analysis.

\subsection{Fracture testing}

All the specimens were conditioned at $23{ }^{\circ} \mathrm{C}$ and $50 \% \mathrm{RH}$ for 24 hours before testing. Tests were carried out at constant displacement rate of $5 \mathrm{~mm} / \mathrm{min}$ and at temperatures of $0,23,40$ and 60 ${ }^{\circ} \mathrm{C}$ on an Instron 1185 dynamometer equipped with a $10 \mathrm{kN}$ load cell and a thermostatic cabinet. The first stage of loading and un-loading (pre-cracking) prior to the actual test, suggested by the standard, was skipped. The reason is that pre-cracking the specimen can cause the development of a small fibre bridging zone whose contribution is not negligible [15]. Therefore, in order to minimize the contribution of fibre-related mechanisms to the fracture toughness at crack initiation, as to highlight the role of the matrix on the delamination toughness of composites, pre-cracking was not performed in the present work. Fracture toughness was measured as the strain energy release rate

$$
G_{I c}=\frac{3 P_{c} u}{2 W(a+|\Delta|)} \frac{1}{N}
$$

where $P_{c}$ is the load, $u$ the displacement, $W$ the specimen width, $a$ the crack length and $\Delta$ and $N$ are correction factors reported in the standard [14]. Fracture toughness was measured both at crack initiation $\left(G_{I c, \text { init }}\right)$ and during the crack propagation stage $\left(G_{I c, p r o p}\right)$ along with crack initiation time $\left(t_{\text {init }}\right)$ and crack propagation speed $(\dot{a})$ following the same approach as in [13]. At least two 
specimens for each temperature were tested, with the only exception of $60{ }^{\circ} \mathrm{C}$ in which only one test, on the composite with toughened matrix, was performed due to experimental problems.

\subsection{Image processing for crack analysis}

Tests were video-recorded with two different high-resolution CCD cameras with a pixel/mm ratio of 20 and 250 respectively. In such a way, it was possible to have, at the same time, a wide view of the whole specimen and a zoom on the crack initiation region where the speckle pattern was applied. Acquisition frequency was 5 frames/s for both cameras. An example of the images taken is given in Fig. 2. Correlated Solutions Vic- $2 \mathrm{D}^{\circledR}$ software was used to perform DIC analysis on the images taken in the initial crack tip region. The Crack Tip Opening Displacement (CTOD) was determined by [16]

$$
C T O D=\left|v_{1}-v_{2}\right|
$$

where $v_{1}$ and $v_{2}$ are the components of the displacement in direction perpendicular to the crack plane measured at the crack tip, on the upper and lower beam respectively. Instead of measuring the relative displacement of two single points close to the crack plane, from the displacement field evaluated with DIC analysis the following procedure was adopted to measure CTOD: the full displacement field $v$ in $y$ direction was measured at $x$ equal to 0 (reference system given in Fig. 2 (b)). An example of the resulting $v$ displacement as a function of $y$ is given in Fig. 3; the two branches of the curve are linearly fitted and the distance between the two intercepts is the $C T O D$. The extrapolation of the value from the linear fitting compensates the noise present in DIC analysis output. CTOD measurements were carried out at crack initiation. The smallest displacement resolvable with this procedure and the parameters adopted was around $5 \mu \mathrm{m}$.

The wide view images were used to measure crack growth and crack opening. A semi-automated procedure implemented in MathWorks MATLAB ${ }^{\circledR}$ software was set-up. For each frame of the video-recordings the crack tip position and the distance between the crack surfaces (at the position corresponding to the initial crack length) were manually individuated. The crack opening, $\delta$, was taken as the distance between the surfaces while the crack growth, $\Delta a$, was simply measured as the distance between the actual crack tip position and its initial one, $a_{0}$ (Fig. 4 (a)).

After testing, the specimens were reloaded up to a load suitable to re-open the bridging zone 
but not to propagate further the crack, and pictures of the bridging zones were taken. A white sheet was put behind the specimens to enhance the contrast between the bridging fibres and the background. The same lighting conditions were adopted for all the specimens. The images were processed with ImageJ software to get grey levels (from 0 to 255) histograms in the area of interest represented by the thick black line in Fig. 4 (a) (iv). An example of the histograms obtained at 23 and $60^{\circ} \mathrm{C}$ is given in Fig. 5. The mean value of a histogram gives an indication of the darkness of the image, hence can be considered proportional, not equal, to the density of fibres in the bridging zone. However, the ratio of the mean values obtained at different temperatures is equal to the ratio of the relevant bridging fibres densities..

\subsection{Fibre bridging cohesive laws determination}

Many authors $[7,8,11,17,18]$ have proposed to derive a cohesive law for fibre bridging adopting an approach based on the application of the $J$-integral [19]. Following this approach $J$ derived along a contour that contains the bridging zone can be expressed as:

$$
J=J_{t i p}+\int_{0}^{\delta} \sigma\left(\delta^{\prime}\right) d \delta^{\prime}
$$

in which $J_{\text {tip }}$ is the critical $J$ value at the crack tip and $\sigma(\delta)$ is the crack surface traction of the bridging zone as a function of the crack opening, $\delta$, at the end of the bridging zone. In eq. (3) and in all the following integrals, the crack opening, $\delta$, is substituted by the corresponding dummy variable of integration, $\delta^{\prime}$.

Unfortunately, it is not easy to directly measure $J$, unless specific test configurations such as DCB specimens loaded with pure bending moments are adopted, as done by some authors to investigate fibre bridging cohesive laws [7, 8]. Energy release rate can be evaluated from other test configurations such as the one adopted in this research, but $J$ is equal to the energy release rate only if the crack growth is self-similar, i.e. during steady-state propagation. When the bridging zone is changing, as it is in the increasing portion of the R-curve, $J$ is not the energy release rate. For this portion of the R-curve Nairn $[11,18]$ has proposed to introduce a corrective term, $W_{B}(\delta)$, which accounts for the recoverable energy in the bridging zone. Then the R-curve, in its increasing 
portion, is given by

$$
R(\delta)=J_{t i p}+\int_{0}^{\delta} \sigma\left(\delta^{\prime}\right) d \delta^{\prime}-W_{B}(\delta)
$$

Following [18] the term $W_{B}(\delta)$ can be expressed as

$$
W_{B}(\delta)=\sigma(\delta) \frac{\delta}{2}
$$

Considering the loading of a specimen as in Fig. 1, the steps depicted in Fig. 4 (a) take place: (i) up to crack initiation the crack opening displacement is equal to 0 , then (ii) as the crack starts to grow a bridging zone develops behind the crack tip until critical crack opening displacement $\left(\delta_{c, F B}\right)$ and length of the bridging zone $\left(\ell_{c, F B}\right)$ are reached (iii). After $\delta_{c, F B}$ is reached, the bridging zone size remains unchanged and simply translates along the beam following the crack tip (iv) and the crack faces left behind are traction free. Fig. 4 (b) shows the stages of evolution of the bridging zone on the R-curve. Stage (iv) is a steady state, where $W_{B}(\delta)$ is zero and $J$-integral coincides with the energy release rate during steady propagation, $G_{I c, p r o p}$. In the same way, $J_{t i p}$ corresponds to the energy release rate at crack initiation, $G_{I c, \text { init }}$. Equation (4) becomes

$$
R(\delta)=G_{I c}(\delta)=G_{I c, \text { init }}+\int_{0}^{\delta} \sigma\left(\delta^{\prime}\right) d \delta^{\prime}-\sigma(\delta) \frac{\delta}{2}
$$

Differentiating equation (6) we obtain:

$$
\frac{\partial G_{I c}(\delta)}{\partial \delta}=\frac{1}{2}\left(\sigma(\delta)-\delta \frac{d \sigma(\delta)}{d \delta}\right)
$$

and by solving the differential equation, with the boundary condition of $\sigma\left(\delta_{c, F B}\right)=0$, the bridging law is finally obtained

$$
\sigma(\delta)=2 \delta \int_{\delta}^{\delta_{c, F B}} \frac{1}{\delta^{\prime 2}} \frac{\partial G_{I c}\left(\delta^{\prime}\right)}{\partial \delta^{\prime}} d \delta^{\prime}
$$

\section{Results and discussion}

Fracture toughness was evaluated for both materials at crack initiation and during crack propagation at different temperatures. The relevant values of fracture initiation time and crack propagation speed were also determined. Results were compared with those already obtained in a previous work [13], in which the pre-cracking procedure suggested in the ISO 15024 standard was 
applied. To this end, data were reduced to a master curve at a reference temperature applying time-temperature superposition, as in [12]: each point was shifted along the logarithmic initiation time or crack speed axis using the shift factors relevant to the matrices previously obtained in [12]. For a discussion on the validity of the approach the reader is referred to $[2,13]$. The results are reported and discussed separately for fracture initiation and fracture propagation.

\subsection{Fracture initiation}

Fig. 6 shows the master curves of $G_{I c \text {,init }}$ vs. $t_{\text {init }}$ for E and EI composites, together with those of the same composites taken from the literature [13]. By comparing the results, it can be observed that $G_{I c}$ is generally lower when pre-cracking is not performed, as found in [20], especially in the case of the EI composite. As mentioned in section 2.2, without pre-cracking the contribution of fibre-related mechanisms is almost negligible resulting in lower values of fracture toughness at crack initiation. To analyse the matrix toughness transfer into the composite, CTOD, which can be considered a measure of the process zone size, was measured as explained in section 2.3. Concerning the E composite, it was not possible to obtain a reliable measurement, since no clear displacement jump at the crack tip, as that of the example of Fig. 3, was observed. This is due to the fact that, as it can be observed in Fig. 7 (a), a micro-cracking mechanism takes place at the crack tip suggesting that fracture occurs at the interface between the matrix and the fibres, probably due to a poor interfacial strength, rather than by the formation of a process zone and crack propagation within the matrix. This agrees with the results in [13]: time dependence of fracture toughness in the composite is different from that of the matrix indicating that the fracture initiation is not dominated by the matrix. Micrographs of the fracture surfaces also show naked fibres with a clean surface, which is the typical feature of a failure dominated by weak fibre interface.

In the case of EI composite, the $C T O D$ was successfully measured at different temperatures and the relevant values are plotted in Fig. 7 (b), along with those previously obtained for the neat EI matrix in [12]. The figure also reports the average thickness of the interlaminar resin-rich layer, measured in [13]. It can be observed that while, in the range examined, the CTOD of the bulk matrix increases with temperature, the $C T O D$ of the composite increases until a threshold value is reached. This value can be taken as the maximum size that the process zone of the matrix can 
reach in the composite and it turned out to be very similar to the thickness of the interlaminar resin-rich layer. Hence, hereby it is experimentally confirmed that, as discussed in the literature, the partial transfer of matrix toughness into the composite occurs when the development of the process zone in the matrix rich layer is hindered by the adjacent fibres.

\subsection{Fracture propagation}

Fig. 8 shows the master curves of $G_{I c, p r o p}$ vs. $\dot{a}$ for E and EI composites, together with those of the same composites from [13]. It can be observed that, in the case of EI composite, the results agree well with those previously obtained, as expected since during steady-state propagation no effect of the initial crack is envisaged. For E composite, instead, $G_{I c \text {,prop }}$ values are slightly higher than the previous ones. This could be due to the different specimen thickness: the specimens of E composite in this work are $5 \mathrm{~mm}$ thick, while in [13] the corresponding thickness was $3 \mathrm{~mm}$. This agrees with $[7,8,9,10]$ where it was found that thicker specimens show higher values of the plateau of the R-curve, i.e. $G_{I c, p r o p}$. As already mentioned, the R-curves turned out to be dependent on the geometry, so they can be considered a specimen property rather than a material one. In many applications, it is obviously more useful to deal with an intrinsic material property, hence the cohesive laws for fibre-bridging were derived, as described in section 2.4. Fig. 9 shows an example of fracture toughness vs. crack opening displacement curve. With the intent of evaluating the derivative (eq. (8)), data were fitted to the following function

$$
G_{I c}=G_{I c, \text { init }}+\Delta G\left(\frac{\delta}{\delta_{c, F B}}\right)^{1 / k}, 0<\delta<\delta_{c, F B}
$$

in which $\Delta G$ is the difference between $G_{I c \text {,prop }}$ and $G_{I c, \text { init }}$. The approach is similar to that described in [8] where $k$ is set equal to 2. In this work, for both composites, at all temperatures considered, the value of $k$ was fairly constant and equal to $2.78 \pm 0.19$. The bridging law can be found simply combining equations (8) and (9):

$$
\sigma(\delta)=\frac{2 \Delta G}{1-2 k}\left[\frac{\delta}{\delta_{c, F B}^{2}}-\frac{1}{\delta}\left(\frac{\delta}{\delta_{c, F B}}\right)^{1 / k}\right] 0<\delta<\delta_{c, F B}
$$

The curves obtained are shown in Fig. 10. While in the case of the E composite no trend with temperature can be observed and, despite the significant scatter, the curves substantially overlap, 
in the case of EI composite there is a clear dependence on temperature, i.e. the stress at any given $\delta$ increases as the temperature increases. In any case, the same result is also evident from Fig. 11 where the values of $\Delta G$ as a function of temperature are plotted: while there is no effect of temperature in the case of E composite, $\Delta G$ increases with temperature in the case of EI composite. The procedure adopted to measure the crack opening displacement from wide view images (section 2.3) gave values, at crack initiation, equal to 0 , instead of values equal to the CTOD measured with DIC analysis. The mismatch was due to the relatively low resolution of wide view images required to observe the whole specimen. This did not affect the derivation of the fibre bridging cohesive laws, since the contribution of matrix to toughness, accounted for by the measurement of $C T O D$, was assumed to be additive. However, a complete cohesive law, in which both the transfer of matrix toughness and the contribution of fibre bridging are considered, can be obtained by translating the origin of the curves by a quantity equal to CTOD and by appending an initial part relevant to matrix contribution. To this end, it was decided to adopt a simple triangular form solution for the cohesive law, evaluating the stress from the fracture toughness at crack initiation and from $C T O D$ [21]. In formulas:

$$
\sigma(\delta)= \begin{cases}\frac{2 G_{I c, \text { init }}}{C^{2} O D^{2}} & 0<\delta<C T O D \\ \frac{2 \Delta G}{1-2 k}\left[\frac{\delta}{\delta_{c, F B}^{2}}-\frac{1}{\delta}\left(\frac{\delta}{\delta_{c, F B}}\right)^{1 / k}\right] & \mathrm{CTOD}<\delta<\delta_{c, F B}\end{cases}
$$

Obviously, this procedure was not applied to the E composite, since CTOD was not measured. The complete curves for EI composite are shown in Fig. 12.

In the case of E composite, as for crack initiation stage, the fracture seems to be governed by interfacial properties that do not change with temperature, rather than by matrix viscoelasticity, as it is possible to derive from the increase of fracture toughness in Fig. 11 and the cohesive laws of Fig. 10 (a).

The EI composite on the other hand, showed a completely different behaviour. The dependence on temperature of the fibre bridging contribution, $\Delta G$, from Fig. 11 is similar to that of the toughness of the matrix on temperature found in [12]. This suggests that the mechanism through which a fibre debonds from the surface and bridges the crack involves mainly fracture within the matrix rather than failure at the fibre/matrix interface. The fractographic analysis reported in [13] agree 
with this hypothesis, as the fibres on the fracture surface appeared covered by the matrix. In order to demonstrate this hypothesis, the model proposed by $[8,22]$ in which the bridging ligament is considered as a cantilever beam under the action of an end load, $P$, was adopted (Fig. 13). The ligaments overcome the fracture resistance $\Gamma_{I}$ and peel away from the crack surface. The displacement, $\delta$, is given by $[8,23]$

$$
\delta=\frac{2 P}{3 E I}\left[\left(\frac{2 w \Gamma_{I}}{P^{2}}-\frac{t^{2}}{2 I G}\right) E I\right]^{3 / 2}+\frac{P t^{2}}{I G}\left[\left(\frac{2 w \Gamma_{I}}{P^{2}}-\frac{t^{2}}{2 I G}\right) E I\right]^{1 / 2}
$$

where $2 t$ and $w$ are the ligament thickness and width respectively, $E$ and $G$ are the Young's modulus and the shear modulus of the ligament respectively and $I$ the second moment of inertia of the cross section of the ligament itself $\left(I=2 w t^{3} / 3\right)$. The stress acting on the crack surfaces is given by the load acting on the single ligament multiplied by the number of ligaments per unit crack surface area, or density of bridging fibres, $N$

$$
\sigma_{L}=P N
$$

By combining eqs. (13) and (12) and neglecting shear deformation, the average bridging stress becomes

$$
\sigma_{L}=c N \Gamma_{I}^{3 / 4} \delta^{-1 / 2}
$$

with

$$
c=\left(\frac{2}{\sqrt{3}}\right)^{3 / 2} w\left(E t^{3}\right)^{1 / 4}
$$

Under the hypothesis that fracture occurs within the matrix the term $\Gamma_{I}$ can be substituted with the fracture toughness at crack initiation, $G_{I c, i n i t}$, being it a measure of the matrix toughness in presence of the fibres.

This model does not predict that tractions in the bridging law become zero when $\delta=\delta_{c, F B}$. As mentioned in [23], eq. (12) or eq. (14) do not consider ligament degradation. This contribution can be integrated in eq. (14) assuming that the fibre density, $N$, is dependent on the opening displacement, $\delta$, with a damage law which is here arbitrarily chosen to be linear:

$$
N(\delta)=N_{0}\left(1-\frac{\delta}{\delta_{c, F B}}\right)
$$


The bridging stress thus becomes

$$
\sigma_{L}=c N_{0} G_{I c, i n i t}^{3 / 4} \delta^{-1 / 2}\left(1-\frac{\delta}{\delta_{c, F B}}\right)
$$

To validate the model the experimental stress (eq. (10)) and the ligament bridging stress (eq. (17)) were compared. To simplify the expressions, the opening displacement was normalized with respect to its critical value, $\delta_{c, F B}$, as

$$
\hat{\delta}=\frac{\delta}{\delta_{c, F B}}
$$

so, the expressions of eqs. (10) and (17) become

$$
\sigma(\hat{\delta})=\frac{2 \Delta G}{1-2 k} \frac{\hat{\delta}^{2}-\hat{\delta}^{1 / k}}{\delta_{c, F B} \hat{\delta}}
$$

and

$$
\sigma_{L}(\hat{\delta})=c N_{0} G_{I c, i n i t}^{3 / 4} \delta_{c, F B}^{-1 / 2} \hat{\delta}^{-1 / 2}(1-\hat{\delta})
$$

respectively. All quantities present in the two equations are known at the different temperatures except for $c$ and $N_{0}$; therefore in Fig. 14 (a) $\sigma c N_{0} / \sigma_{L}$ is plotted as a function of $\hat{\delta}$. Apart from an initial transient at lower values of $\hat{\delta}$, the curves show a fairly constant trend; the average value can be thought to correspond to $c N_{0}$. The dependence of the ratio on $\hat{\delta}$, for small values of $\hat{\delta}$ is probably caused by the approximation introduced neglecting the shear terms in eq. (12), and on the whole range the curves probably are affected by the arbitrariness of the damage function introduced in eq. (16). A further verification of the validity of the model was made by comparing the temperature dependence of the density of bridging fibre $N_{0}$ from eqs. (19) and (20) and comparing it with directly measured values of $N$. Considering that, as mentioned above, $k$ in eq. (19) was found to be temperature independent and the term $c$ in eq. (20) does not depend on either temperature or $\hat{\delta}$, since it is only related to the geometry of the ligament and to its modulus, which is taken as a constant equal to the fibre modulus in the first approximation, the two equations for the stress (eqs. (19) and (20)) were rewritten as the product of three terms: the first, $a_{1}$ or $c$, is constant, the second, $f_{1}$ or $f_{2}$, is a function of temperature only and the third, $g_{1}$ or $g_{2}$, is a function of the opening displacement only:

$$
\sigma(T, \hat{\delta})=\frac{2}{1-2 k} \cdot \frac{\Delta G(T)}{\delta_{c, F B}(T)} \cdot\left(\hat{\delta}-\hat{\delta}^{\frac{1}{k}-1}\right)=a_{1} \cdot f_{1}(T) \cdot g_{1}(\hat{\delta})
$$


and

$$
\sigma_{L}(T, \hat{\delta})=c \cdot \frac{N_{0}(T) G_{I c, i n i t}^{3 / 4}(T)}{\delta_{c, F B}^{1 / 2}} \cdot \hat{\delta}^{-1 / 2}(1-\hat{\delta})=c \cdot f_{2}(T) \cdot g_{2}(\hat{\delta})
$$

respectively. The only terms still unknown are $c$ and $N_{0}$, but since the variables were separated, it is possible to normalize eqs. (21) and (22) with respect to a reference temperature, $T_{0}$. In formulas:

$$
\sigma^{*}\left(T, T_{0}\right)=\frac{\sigma(T)}{\sigma\left(T_{0}\right)}=\frac{a_{1}}{a_{1}} \frac{f_{1}(T)}{f_{1}\left(T_{0}\right)} \frac{g_{1}(\hat{\delta})}{g_{1}(\hat{\delta})}=\frac{f_{1}(T)}{f_{1}\left(T_{0}\right)}
$$

and, similarly

$$
\sigma_{L}^{*}\left(T, T_{0}\right)=\frac{f_{2}(T)}{f_{2}\left(T_{0}\right)}
$$

Once the stresses are normalized with respect to temperature, they can be equated and the fibre density, $N_{0}$, can be isolated, obtaining

$$
\frac{N_{0}(T)}{N_{0}\left(T_{0}\right)}=\frac{N_{T}}{N_{T_{0}}}=\frac{\Delta G(T)}{\Delta G\left(T_{0}\right)}\left(\frac{G_{I c, \text { init }}\left(T_{0}\right)}{G_{I c, \text { init }}(T)}\right)^{3 / 4}\left(\frac{\delta_{c, F B}\left(T_{0}\right)}{\delta_{c, F B}(T)}\right)^{1 / 2}
$$

The critical value of the opening displacement, $\delta_{c, F B}$, was found to be almost constant and there was not a clear dependence on temperature, at least in the range investigated. However, since the standard deviation from the mean value was not small as in the case of the term $k$, the values measured in each test, instead of the average value, were used to evaluate the ratio $N_{T} / N_{T_{0}}$. This ratio, setting as reference temperature, $T_{0}, 23{ }^{\circ} \mathrm{C}$, is shown in Fig. 14 (b) (crosses). A measure of the density of bridging fibres was also independently carried out as explained in sec. 2.3. The results, normalized with respect to the value obtained at $23{ }^{\circ} \mathrm{C}$, are also reported in Fig. 14 (b) (circles). It can be observed the good agreement with the values derived from the cohesive laws and bridging ligament peeling model, thus confirming its applicability.

\section{Conclusions}

The interlaminar fracture behaviour of continuous fibre composite materials prepared using unidirectional carbon fibres and two thermoplastic acrylic resins, one plain and one toughened, was investigated at different temperatures to better understand matrix toughness transfer and fibre bridging contribution. Results are discussed in comparison with those previously obtained for 
the same composites [13] and the relevant matrices [12]. For composites prepared with the plain resin, the fracture toughness turned out to be dominated by the poor interfacial strength between the matrix and the fibres both at crack initiation and during propagation. The fracture toughness was almost constant for all testing conditions, and no significant contribution of fibre bridging to the fracture behaviour of the composite was observed, as expected, due to the poor interfacial strength.

On the contrary, the fibre/matrix adhesion was improved by the use of the toughened resin and the fracture toughness of this material had the same dependence on temperature as the matrix. From this research, the following conclusions could be drawn:

- It was verified, from the optical measurement of crack tip opening displacement, that the process zone of the matrix in the composite can reach a maximum size which is very close to the thickness of the interlaminar matrix-rich layer in which the crack propagates.

- Fibre bridging was modelled by making use of experimentally determined cohesive laws and a model from the literature which considers fibre bridging as the peeling of a ligament across the crack. A good agreement between the two approaches was found confirming that the fracture process occurs within the matrix rather at the fibre interface.

\section{Acknowledgements}

Authors would like to thank Arkema Company and the Groupement de Recherche du Lacq, France for the materials and the support provided.

\section{References}

[1] W. Jordan, W. Bradley, R. Moulton, Relating Resin Mechanical Properties to Composite Delamination Fracture Toughness, J. Compos. Mater. 23 (9) (1989) 923-943, doi:10.1177/002199838902300904.

[2] R. Frassine, A. Pavan, Viscoelastic effects on the interlaminar fracture behaviour of thermoplastic matrix composites: I. Rate and temperature dependence in unidirectional PEI/carbon-fibre laminates, Compos. Sci. Technol. 54 (2) (1995) 193-200, doi:10.1016/0266-3538(95)00051-8. 
[3] K. Friedrich, R. Walter, L. A. Carlsson, A. J. Smiley, J. W. Gillespie, Mechanisms for rate effects on interlaminar fracture toughness of carbon/epoxy and carbon/PEEK composites, J. Mater. Sci. 24 (9) (1989) 3387-3398, doi: 10.1007/BF01139070.

[4] R. Frassine, M. Rink, A. Pavan, Viscoelastic effects on the interlaminar fracture behaviour of thermoplastic matrix composites: II. Rate and temperature dependence in unidirectional PEEK/carbon-fibre laminates, Compos. Sci. Technol. 56 (11) (1996) 1253-1260, doi:10.1016/S0266-3538(96)00084-X.

[5] N. A. Siddiqui, R. S. Woo, J.-K. Kim, C. C. Leung, A. Munir, Mode I interlaminar fracture behavior and mechanical properties of CFRPs with nanoclay-filled epoxy matrix, Compos. Part A Appl. Sci. Manuf. 38 (2) (2007) 449-460, doi:10.1016/j.compositesa.2006.03.001.

[6] S. A. Ngah, A. C. Taylor, Toughening performance of glass fibre composites with coreshell rubber and silica nanoparticle modified matrices, Compos. Part A Appl. Sci. Manuf. 80 (2016) 292-303, doi: 10.1016/j.compositesa.2015.10.036.

[7] Z. Suo, G. Bao, B. Fan, Delamination R-curve phenomena due to damage, J. Mech. Phys. Solids 40 (1) (1992) 1-16, doi:10.1016/0022-5096(92)90198-B.

[8] B. F. Sørensen, T. K. Jacobsen, Large-scale bridging in composites: R-curves and bridging laws, Compos. Part A Appl. Sci. Manuf. 29 (11) (1998) 1443-1451, doi:10.1016/S1359-835X(98)00025-6.

[9] B. D. Manshadi, A. P. Vassilopoulos, J. Botsis, A combined experimental/numerical study of the scaling effects on mode I delamination of GFRP, Compos. Sci. Technol. 83 (2013) 32-39, doi: 10.1016/j.compscitech.2013.04.016.

[10] E. Farmand-Ashtiani, J. Cugnoni, J. Botsis, Specimen thickness dependence of large scale fiber bridging in mode I interlaminar fracture of carbon epoxy composite, Int. J. Solids Struct. 55 (2015) 58-65, doi: 10.1016/j.ijsolstr.2014.03.031.

[11] B. Mirzaei, A. Sinha, J. A. Nairn, Measuring and modeling fiber bridging: Application to wood and wood composites exposed to moisture cycling, Compos. Sci. Technol. 128 (2016) 65-74, doi: 10.1016/j.compscitech.2016.03.017.

[12] T. Pini, F. Briatico-Vangosa, R. Frassine, M. Rink, Fracture toughness of acrylic resins: Viscoelastic effects and deformation mechanisms, Polym. Eng. Sci. doi:10.1002/pen.24583.

[13] T. Pini, F. Caimmi, F. Briatico-Vangosa, R. Frassine, M. Rink, Fracture initiation and propagation in unidirectional CF composites based on thermoplastic acrylic resins, Eng. Fract. Mech. 184 (2017) 51-58, doi: 10.1016/j.engfracmech.2017.08.023.

[14] ISO 15024, Fibre-reinforced plastic composites - Determination of mode I interlaminar fracture toughness, GIc, for unidirectionally reinforced materials, Tech. Rep. 15024, International Organization for Standardization, Geneva, CH, 2001.

[15] D. Stevanovic, P.-Y. Jar, S. Kalyanasundaram, A. Lowe, On crack-initiation conditions for mode I and mode 
II delamination testing of composite materials, Compos. Sci. and Technology 60 (9) (2000) 1879-1887, doi: 10.1016/S0266-3538(00)00080-4.

[16] J. Xavier, M. Oliveira, J. J. L. Morais, M. F. S. F. de Moura, Determining mode I cohesive law of Pinus pinaster by coupling double cantilever beam test with digital image correlation, Frat. ed Integrità Strutt. 31 (2015) 13-22, doi:10.3221/IGF-ESIS.31.02.

[17] G. Bao, Z. Suo, Remarks on Crack-Bridging Concepts, Appl. Mech. Rev. 45 (8) (1992) 355-366, doi: 10.1115/1.3119764.

[18] J. A. Nairn, Analytical and numerical modeling of R curves for cracks with bridging zones, Int. J. Frac. 155 (2) (2009) 155-167, doi:10.1007/s10704-009-9338-3.

[19] J. R. Rice, A Path Independent Integral and the Approximate Analysis of Strain Concentration by Notches and Cracks, J. Appl. Mech. 35 (1968) 379-386, doi:10.1115/1.3601206.

[20] S. Hashemi, A. Kinloch, J. Williams, The Effects of Geometry, Rate and Temperature on the Mode I, Mode II and Mixed-Mode I/II Interlaminar Fracture of Carbon-Fibre/Poly(ether-ether ketone) Composites, J. Compos. Mater. 24 (9) (1990) 918-956, doi:10.1177/002199839002400902.

[21] J. Williams, H. Hadavinia, Analytical solutions for cohesive zone models, J. Mech. Phys. Solids 50 (2002) 809-825, doi:10.1016/S0022-5096(01)00095-3.

[22] B. F. Sørensen, T. K. Jacobsen, Determination of cohesive laws by the J integral approach, Eng. Fract. Mech. 70 (14) (2003) 1841-1858, doi:10.1016/S0013-7944(03)00127-9.

[23] S. Spearing, A. Evans, The role of fiber bridging in the delamination resistance of fiber-reinforced composites, Acta Metall. Mater. 40 (9) (1992) 2191-2199, doi:10.1016/0956-7151(92)90137-4.

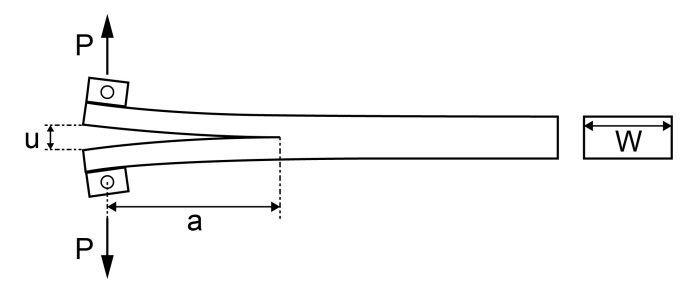

Figure 1: Double cantilever beam test specimen. 


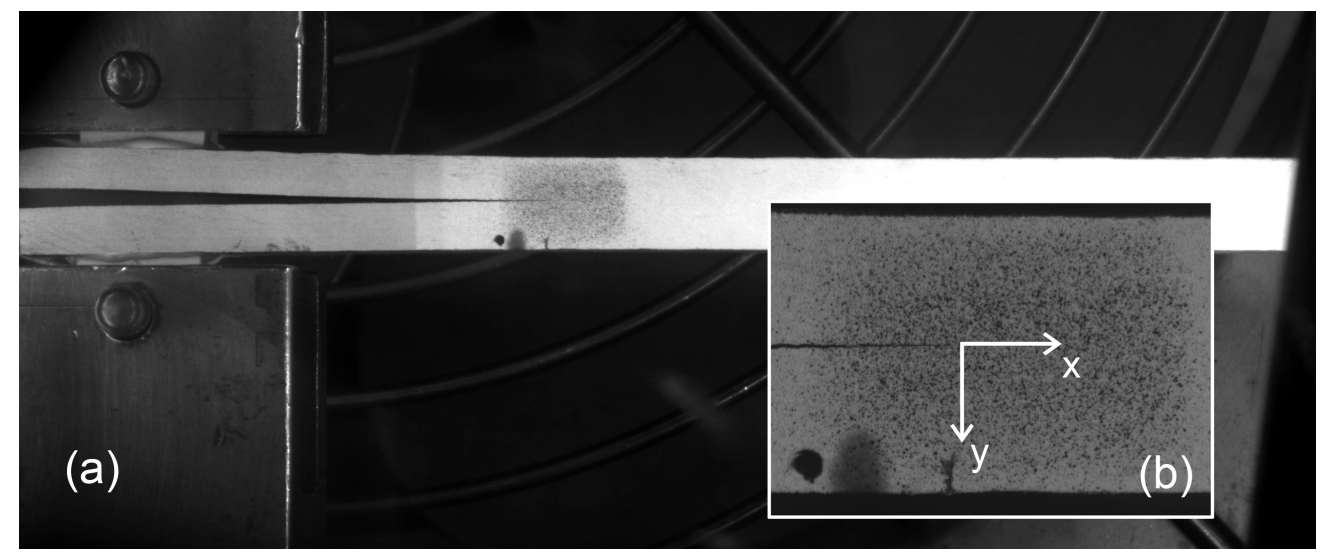

Figure 2: (a) Overall view of DCB specimen during test. In the white box (b) the zoom on the initial crack tip region.

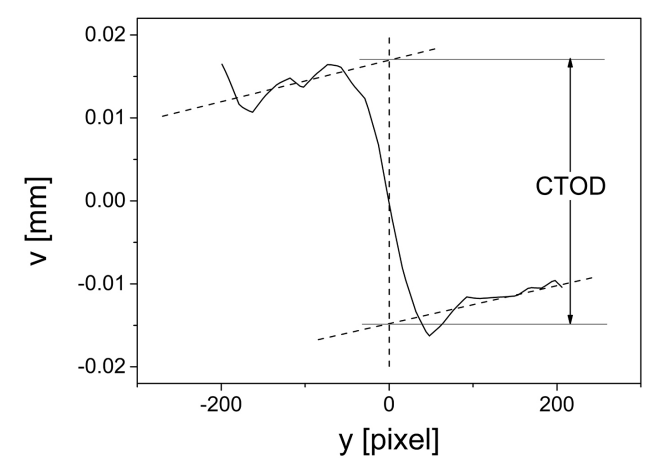

Figure 3: Measurement method for CTOD.
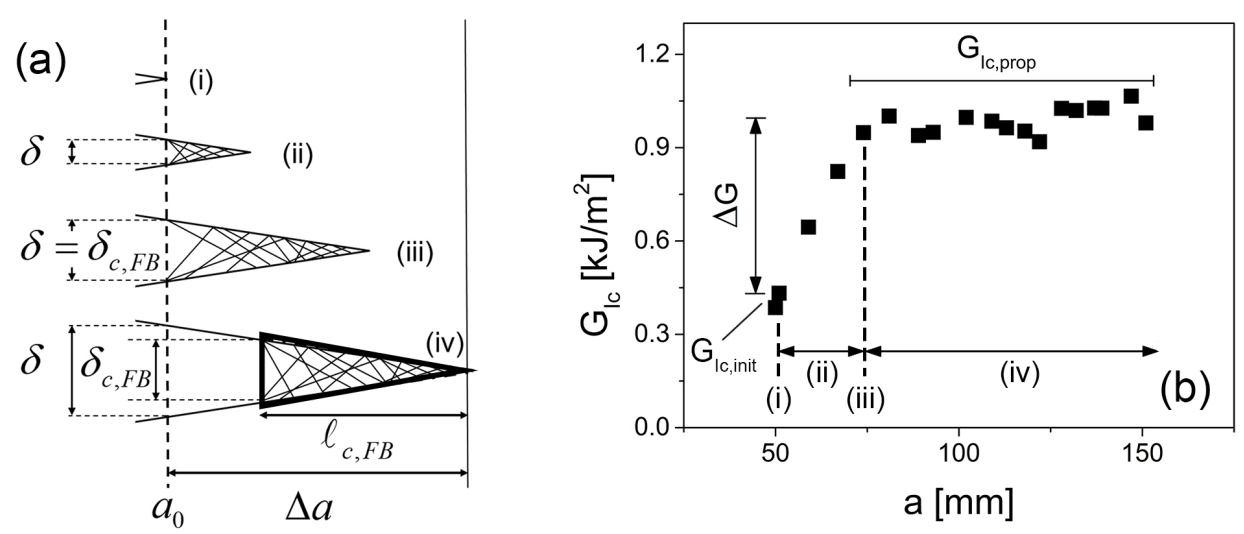

Figure 4: (a) Schematic of the evolution stages of fibre bridging zone. (b) Example of an R-curve with the relevant evolution of fibre bridging. 


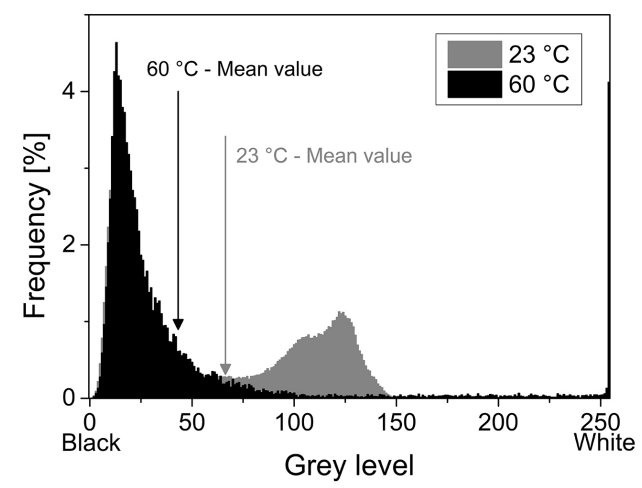

Figure 5: Grey levels histograms of the bridging zone of the specimens tested at 23 and $60{ }^{\circ} \mathrm{C}$
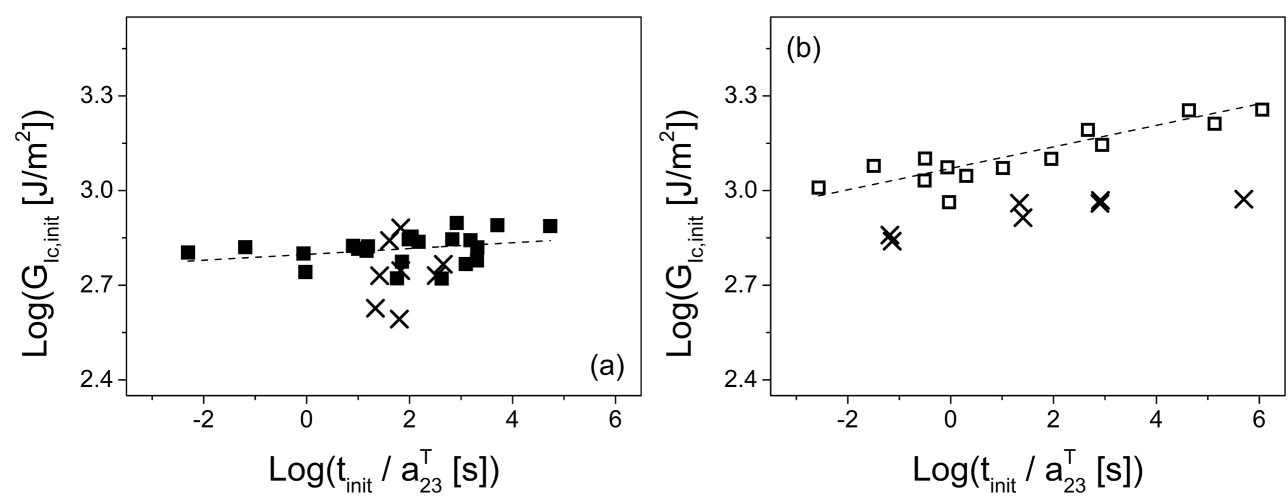

Figure 6: Fracture toughness at crack initiation, $G_{I c, \text { init }}$, vs. crack initiation time, $t_{\text {init }}$, master curves for E (a) and EI composite (b). Data from present work (crosses), and from [13] (squares).
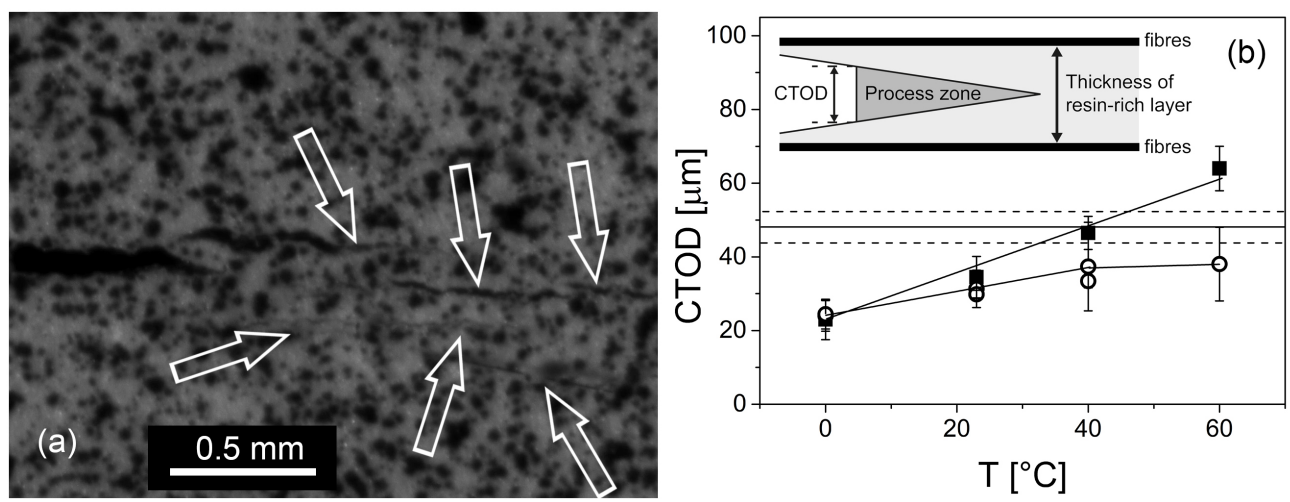

Figure 7: (a) Crack initiation region for E composite. The white arrows point to the side cracks that were observed. (b) Crack Tip Opening Displacement $(C T O D)$ as a function of temperature for EI composite (circles) and matrix (squares). The horizontal continuous and dashed lines represent the average thickness of the interlaminar resin-rich layer and the relevant standard deviation. 

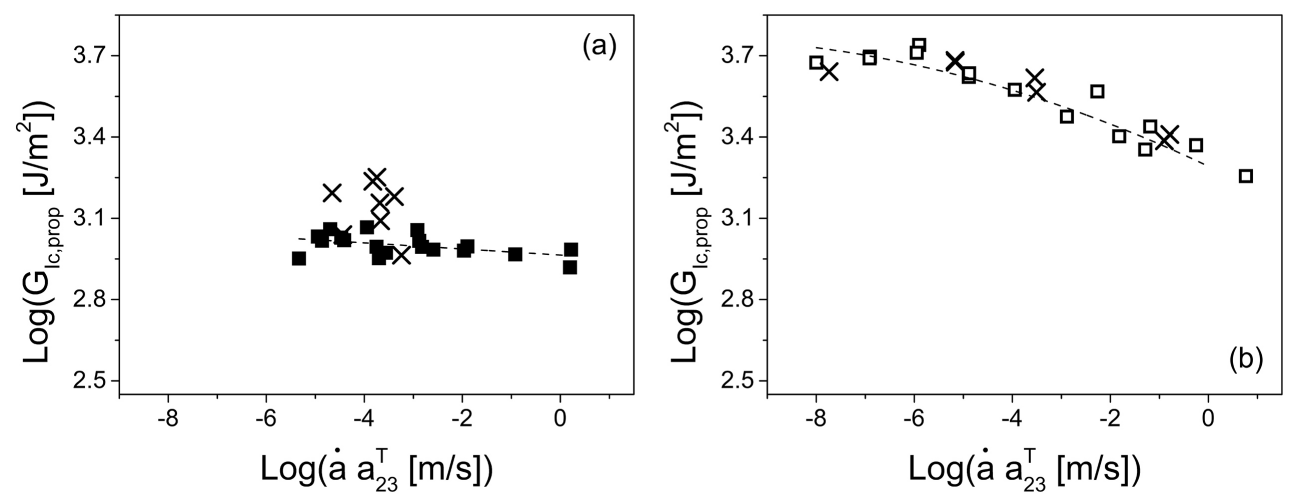

Figure 8: Fracture toughness, $G_{I c, p r o p}$ vs. crack propagation speed, $\dot{a}$, master curves for E (a) and EI composite (b). Data from present work (crosses) and from [13] (squares).

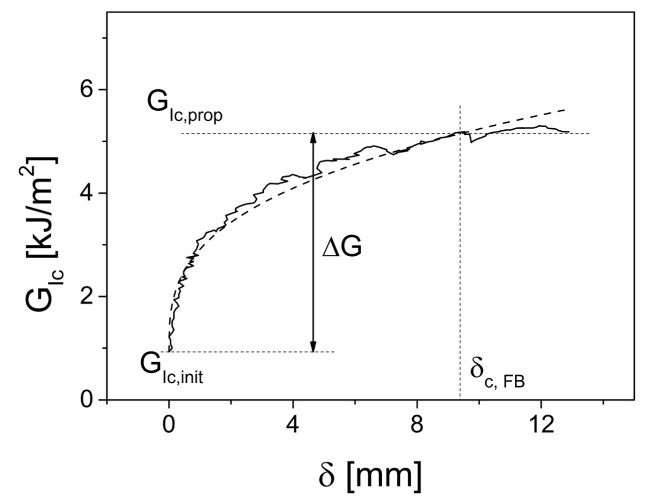

Figure 9: Example of a fracture toughness, $G_{I c}$, vs. crack opening displacement, $\delta$, plot. Solid line is experimental data, dashed line is the fitting function from equation (9).
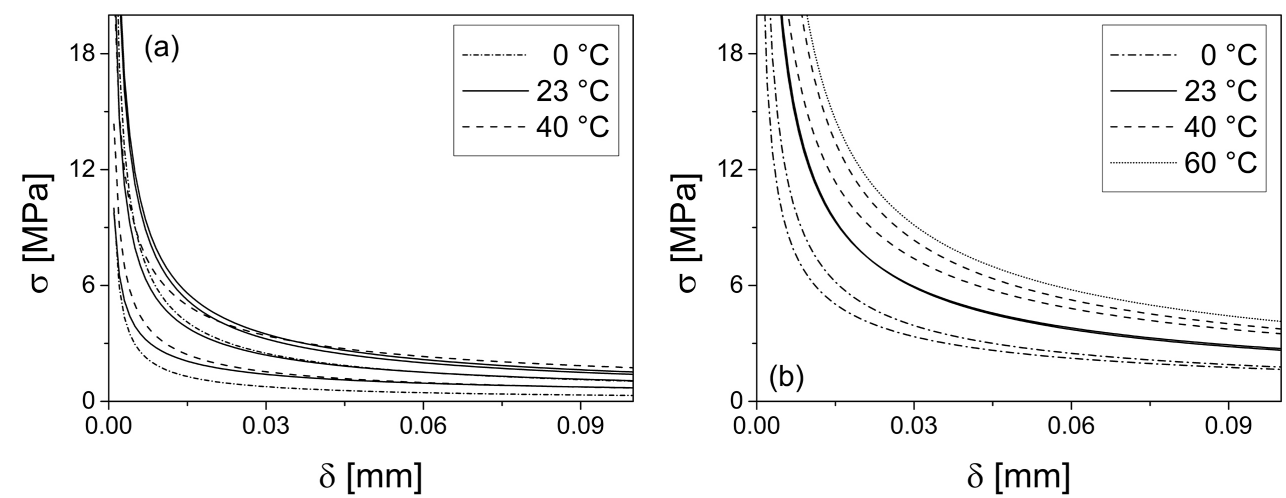

Figure 10: Cohesive laws, stress, $\sigma$, vs. crack opening displacement, $\delta$, for $\mathrm{E}$ (a) and EI composite (b) at different temperatures. 


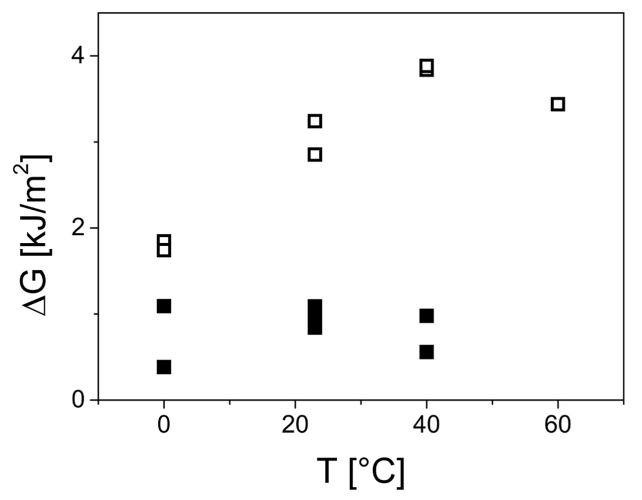

Figure 11: Fracture toughness difference between crack propagation and initiation, $\Delta G$, vs. temperature, $T$, for $\mathrm{E}$ (solid squares) and EI composite (open squares).

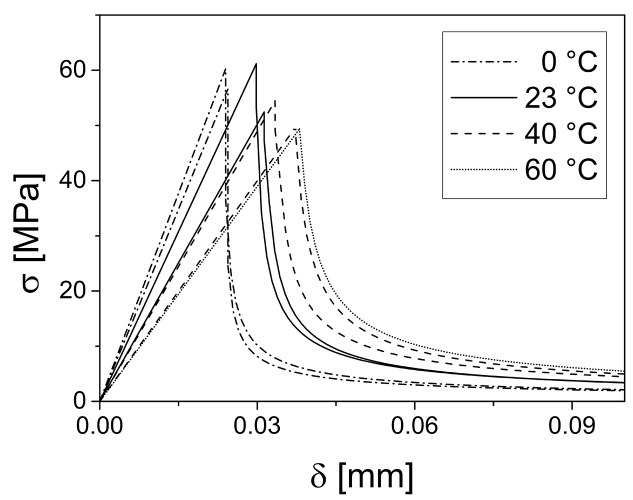

Figure 12: Complete cohesive laws, stress, $\sigma$, vs. crack opening displacement, $\delta$, for EI composite at different temperatures.

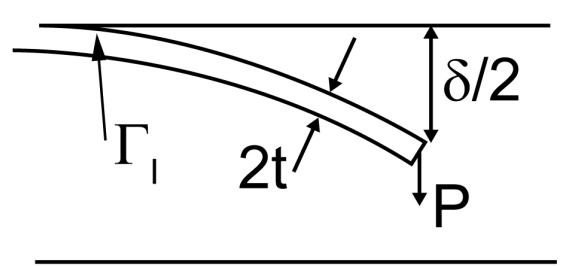

Figure 13: Model of bridging ligament. 

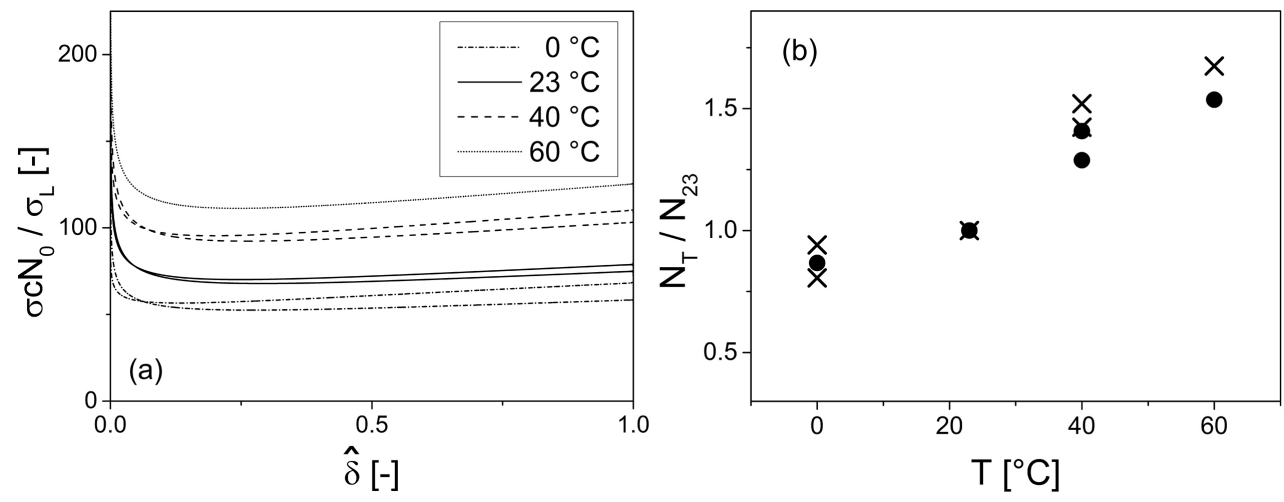

Figure 14: (a) Ratio of bridging stresses from R-curves (eq. (19)) and from bridging ligament model (eq. 20) multiplied by $c N_{0}$ as a function of the normalized opening displacement, $\hat{\delta}$. (b) Normalized bridging fibres density, $N_{T} / N_{23}$, vs. temperature, $T$, from equation (25) (crosses) and from direct measurements (circles). 


\section{List of Figures}

1 Double cantilever beam test specimen. . . . . . . . . . . . . . . . 17

2 (a) Overall view of DCB specimen during test. In the white box (b) the zoom on the initial crack tip region. . . . . . . . . . . . . . . . . 18

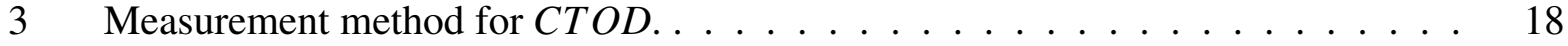

4 (a) Schematic of the evolution stages of fibre bridging zone. (b) Example of an R-curve with the relevant evolution of fibre bridging. . . . . . . . . . . . 18

5 Grey levels histograms of the bridging zone of the specimens tested at 23 and $60{ }^{\circ} \mathrm{C} 19$

6 Fracture toughness at crack initiation, $G_{I c, \text { init }}$, vs. crack initiation time, $t_{\text {init }}$, master curves for E (a) and EI composite (b). Data from present work (crosses), and from $[13]$ (squares). . . . . . . . . . . . . . . . . . . .

7 (a) Crack initiation region for E composite. The white arrows point to the side cracks that were observed. (b) Crack Tip Opening Displacement (CTOD) as a function of temperature for EI composite (circles) and matrix (squares). The horizontal continuous and dashed lines represent the average thickness of the interlaminar resin-rich layer and the relevant standard deviation. . . . . . . . . .

8 Fracture toughness, $G_{I c, p r o p}$ vs. crack propagation speed, $\dot{a}$, master curves for E (a) and EI composite (b). Data from present work (crosses) and from [13] (squares). 20

9 Example of a fracture toughness, $G_{I c}$, vs. crack opening displacement, $\delta$, plot. Solid line is experimental data, dashed line is the fitting function from equation (9). 20

10 Cohesive laws, stress, $\sigma$, vs. crack opening displacement, $\delta$, for E (a) and EI composite $(b)$ at different temperatures. . . . . . . . . . . . . . . 20

11 Fracture toughness difference between crack propagation and initiation, $\Delta G$, vs. temperature, $T$, for E (solid squares) and EI composite (open squares). . . . . . .

12 Complete cohesive laws, stress, $\sigma$, vs. crack opening displacement, $\delta$, for EI composite at different temperatures. . . . . . . . . . . . . . . . . 21

13 Model of bridging ligament. . . . . . . . . . . . . . . . . 21 
14 (a) Ratio of bridging stresses from R-curves (eq. (19)) and from bridging ligament model (eq. 20) multiplied by $c N_{0}$ as a function of the normalized opening displacement, $\hat{\delta}$. (b) Normalized bridging fibres density, $N_{T} / N_{23}$, vs. temperature, $T$, from equation (25) (crosses) and from direct measurements (circles). . . . . . . . . . 22 